Brit. Heart F., 1969, 31, 531.

\title{
An Electrocardiogram Reporting Service: its Role and its Risks
}

\section{DAVID SHORT*}

There is a growing demand by family doctors for the provision of an electrocardiographic service and report without clinical examination for patients with suspected coronary heart disease (British Medical Association, 1968). This demand reflects an important need and requires serious consideration, for the diagnosis of coronary heart disease is one of the most difficult problems which the family doctor faces, and he needs an objective test to help him. Furthermore, he wants to be able to make the diagnosis himself without calling in another doctor to see the patient. But is an electrocardiogram reporting service a satisfactory solution to this problem? Does it lead to accurate diagnosis ? This is the vital question. Several hospitals are already providing electrocardiographic clinics, and some have reported satisfaction on the part of both family doctors and those providing the service (Seymour, Conway, and Bridger, 1968; Lorimer and Kennedy, 1968). On the other hand, doubts have been expressed about the value of such an arrangement on two grounds: first that a high proportion of the cardiograms of patients with suspected coronary disease show non-specific abnormalities; and second that even where the tracing does indicate the presence of coronary heart disease, there is frequently no evidence that this is recent and the explanation of the patient's symptoms (Short, 1969).

\section{Classical Patterns of INFARCTION AND theIR APPLICABILITY}

The first question which requires consideration is which cardiographic patterns may be accepted as evidence of coronary heart disease. A World Health Organization Expert Committee on Cardiovascular Diseases and Hypertension (1959) studied

\footnotetext{
* Address: Royal Infirmary, Aberdeen.
}

this matter in order to provide a basis for epidemiological studies. They proposed two sets of criteria: one for the diagnosis of "very probable" myocardial infarction, and the other for "possible" infarction. The criteria for the diagnosis of "very probable" infarction were $Q$ waves of a specified size and ST elevation with a characteristic evolution. These may be considered the classical criteria of infarction. A number of other cardiographic abnormalities commonly found in coronary heart disease were listed as being consistent with "possible" infarction. These included transient raising or lowering of the ST segment, symmetrical negative $T$ waves from V3 to V6, small $Q$ or $Q S$ waves, conspicuous left axis deviation with a deep SII, SIII, or rr'II, right axis deviation associated with a hypertrophied left ventricle, low voltage in a patient with a hypertrophied heart of hypertension, right or left bundle-branch block with additional $Q$ waves, high early $R$ waves in VI, TIII greater than TI, and positive effort tests.

It will be noted first that the W.H.O. Committee did not list any abnormalities as being absolutely diagnostic of infarction, and secondly that the criteria they laid down for "very.probable" infarction are those associated with large transmural lesions. To many, this may appear to be an extremely conservative attitude; yet it is one to which the majority of authorities on electrocardiography would still subscribe. It is dictated by the knowledge that the cardiographic abnormalities found in coronary heart disease may be simulated by non-coronary disease; and by the experience that a mistaken diagnosis of coronary disease may have serious repercussions on a patient's life and livelihood.

Nevertheless, it is generally recognized that these classical criteria are frequently lacking in patients with proven infarction. This has been established by a number of studies correlating electrocardiograms recorded shortly before death with the state 
of the heart at subsequent necropsy. For example, Woods, Laurie, and Smith (1963) analysed a necropsy series of 122 cases with ischaemic myocardial damage, all of whom had at least one adequate 12lead record; all cases with acute damage had a tracing taken within 48 hours of death. Of the 122 patients, 95 had major confluent infarction, and the remainder had patchy ischaemic fibrosis. All but one of the cardiograms showed some abnormality, but only 48 per cent fulfilled the W.H.O. criteria of "very probable" infarction; this was most frequent in acute infarcts $(82 \%)$ and least frequent in single healed infarcts (27\%). Mitchell and Schwartz (1963) analysed the electrocardiograms in 56 patients with massive cardiac necrosis or massive scarring, and found that 47 fulfilled the classical criteria of infarction, but 4 showed only doubtful ST and T wave abnormalities and 5 only left bundle-branch block. Skjaeggestad and Molne (1966) analysed a necropsy series of 134 patients with healed infarction, all of whom had had a standard 12-lead tracing recorded within a month of death. Though the cardiogram was abnormal in all but 3 patients, classical patterns were found in only 35 per cent. Sodi-Pallares et al. (1963), in a necropsy study of 106 patients, found that infarction of the free wall of the left ventricle was poorly recognized, especially if the infarction was subendocardial or high lateral in position. Cook, Edwards, and Pruitt (1958a, b) showed that small or moderate subendocardial infarcts rarely exhibited the classical criteria of infarction and even large subendocardial infarcts frequently failed to do so.

In most of the studies summarized above, the infarcts were large and fatal. The type of case which presents a problem to the family doctor is the one in which the infarct is small or subacute. Since major infarcts frequently fail to show classical cardiographic abnormalities, it is not surprising that small infarcts do so less commonly. This was shown by a study of 77 patients whose symptoms were regarded by their family doctors as too slight or indefinite to warrant admission to hospital (Short, 1968). In each case, a diagnosis of myocardial infarction was established either by serial cardiographic changes, unequivocal serum enzyme changes associated with a classical clinical picture, or by necropsy. Only 28 of the 77 patients had abnormalities in their initial records which fulfilled the W.H.O. criteria of "very probable" infarction; 19 had ST elevation, and $9 \mathrm{ab}$ normal $Q$ and $T$ waves. Of the remainder, $17 \mathrm{had}$ T wave inversion, 6 had ST depression of more than $0.5 \mathrm{~mm}$., and 7 showed a pattern of pure left ventricular hypertrophy, while 19 showed no abnormality classifiable under the Minnesota
Code*, though 10 of these showed slight ST depression and/or $\mathrm{T}$ wave flattening.

Another group of cases in which classical cardiographic appearances are lacking is those with healed infarcts. Burns-Cox (1967) found that four years after a single infarction as many as 20 per cent of patients had an electrocardiogram which showed no abnormality codifiable under the Minnesota Code. In patients with slight coronary attacks, cardiographic recovery is the rule (Papp and Smith, 1951).

We may sum up the reliability of the W.H.O. criteria of "very probable" infarction by saying that though they are almost never seen in the absence of infarction, they are frequently lacking in cases of proven infarction, particularly when the infarct is small, situated in the subendocardium, or the lateral part of the left ventricle; when there are superimposed patterns of bundle-branch block or left ventricular hypertrophy; or when the electrocardiogram is recorded early in the development of the infarct or long after it has healed. The cardiogram of recognizable infarction develops out of normality and regresses towards normality, so that at various stages it must pass through borderline patterns; and if it be a small infarct it may never progress beyond these stages.

\section{Other Electrocardiographic ABNormalities AND THEIR RELIABILITY}

Since the W.H.O. criteria of "very probable" myocardial infarction are lacking in the case of many infarcts, even acute ones, it is clear that any electrocardiogram reporting service which relied entirely on these criteria would be a highly unsatisfactory and dangerous institution. We must therefore consider whether we can broaden our electrocardiographic criteria, and, if so, what safeguards are needed to avoid the erroneous diagnosis of infarction.

Evans and McRae (1952) have drawn attention to a number of minor cardiographic abnormalities commonly associated with coronary heart disease, most of which are too slight to be recognized by the Minnesota Code. The most frequent and clearly recognizable of these changes is "plane" ST depression-a minor degree of horizontal ST depression, or even a horizontal prolongation of the ST segment without actual depression below the

\footnotetext{
* The Minnesota Code (Blackburn et al., 1960) is a widely recognized definition and classification of electrocardiographic abnormalities. According to this system, ST elevation must be at least $1.0 \mathrm{~mm}$. to be recognized as abnormal, ST depression $0.5 \mathrm{~mm}$. if horizontal or downward sloping, and $1.0 \mathrm{~mm}$. if of a junctional type. $T$ waves are recognized as abnormal if they are flat or diphasic, but not if they are merely low.
} 
level of the PR segment. They also described downward sloping or sagging ST depression as evidence of coronary heart disease. Other "lesser signs of infarction" described by Evans (1965) include inversion or abrupt descent of the terminal part of the $T$ wave, a $T$ wave which is low or blunt topped, notching of the $R$ wave in a left praecordial lead in which the complex is of the $q R$ type, $T U$ segment depression, $U$ wave inversion, and the appearance of a $Q$ wave or ST depression in lead III on taking a deep breath.

A number of other cardiographic abnormalities have been described in coronary heart disease. The abnormalities listed by the W.H.O. expert committee as consistent with "possible" infarction (see above) are frequently found in the acute or healing stages of infarction. An increased $T$ wave voltage is sometimes seen in acute infarction, but this cannot be recognized as definitely abnormal on a single record. A rare sign of infarction is a $Q$ wave in an extrasystole occurring in a lead where such a wave would be abnormal if it were found in a normally conducted beat (Bisteni, Medrano, and Sodi-Pallares, 1961).

In terms of frequency, however, the most important of the non-classical signs of coronary heart disease are minor degrees of ST depression and deformity of the $T$ wave. Indeed, these are often the only cardiographic abnormalities found in small infarcts (Papp and Smith, 1951; Cook et al., 1958b; Short, 1968). Evans (1965) claims that if his signs are recognized, the cardiogram is invariably found to be abnormal in coronary heart disease-even years before symptoms appear-so that a strictly normal tracing excludes this diagnosis. Most cardiologists at the present time, however, would regard it as dangerous to exclude coronary disease on the basis of a single normal tracing.

Though the abnormalities described above undoubtedly show a close correlation with myocardial infarction, it is essential to know how specific they are. Are they found in non-coronary conditions? Are they ever found in health? Since ST depression and abnormalities of the $T$ wave are the features most closely and commonly associated with coronary heart disease, we will confine our attention to these.

Most cardiologists consider that the slight ST and $T$ wave abnormalities described above are not specific for coronary disease. Certainly, similar appearances have been described in a wide variety of non-coronary diseases, and even in physiological conditions. The most frequent source of confusion is left ventricular hypertrophy (Short, 1969). Other diseases causing similar electrocardiographic abnormalities include myocarditis, alcoholic cardio- myopathy, and hypothyroidism. ST and T wave depression may also be seen in pregnancy, in patients taking digoxin (or other cardiac glycosides) or one of the tricyclic anti-depressive drugs (such as imipramine and amitriptyline), as well as in a variety of circumstances ranging from drinking iced water to driving through rush-hour traffic (Table).

Since $S T$ and $T$ wave abnormalities resembling those of coronary heart disease are found in so many other conditions, the question arises whether they have any value as evidence of coronary heart disease. Many authorities think not. Friedberg (1966), for example, strongly criticizes what he calls "exaggerated interpretations of non-specific changes", regarding these as a cause of over-diagnosis of coronary heart disease and resulting unwarranted cardiac invalidism. In his view, " $S-T$ depression of less than $0.5 \mathrm{~mm}$. and $T$ wave inversion of less than $1 \mathrm{~mm}$. are not significant or at least not due to myocardial ischaemia".

Nevertheless we need to be realistic, and to ask how commonly these abnormalities are found in noncoronary conditions and to what extent they present a serious problem in differential diagnosis in the clinical situation. Friedberg and Zager (1961) analysed 209 electrocardiograms showing nonspecific ST and T wave changes out of a series of 1000 in-patient tracings submitted for reporting.

TABLE

SOME NON-CORONARY CAUSES OF ST AND T WAVE ABNORMALITIES

(1) Physiological
Post-prandial
Iced drinks
Orthostatic
Driving motor car
Valsalva manoeuvre
Adolescence
Pregnancy
Racial
"Suspended heart"
(2) Environmental
High altitude
Hypoxia
Cold
(3) Pharmacological and Experimental
Tobacco smoking
Cardiac glycosides
Tricyclic anti-depressant drugs
Atropine
Catecholamines
Sympathetic stimulation
(4) Pathological
Cardiac
Ventricular hypertrophy
Myocarditis
Pericarditis
Alcoholism
Radiation injury
Extracardiac
Electrolyte disturbance, e.g. hypokalaemia
Metabolic diseases, e.g. hypothyroidism, cortisol deficiency
Intracranial disease
Acute pancreatitis
Electrocardiograph machine with inadequate low-frequency
response
(5) Artefactual 
Of the 209 patients, 46 were found to be on some form of digitalis and 57 had had recent cardiac pain; this left 106 to be explained. Coronary heart disease and/or hypertension were thought to be responsible for 27 of these, intracranial disease for 10, and rheumatic heart disease for 5. Pulmonary embolism, post-operative shock, pericarditis, anaemia, cor pulmonale, or electrolyte abnormalities were regarded as the probable explanation in 15 more; but even after diligent investigation, 49 still remained unexplained. Thus, though many different factors were found to be capable of causing similar ST and $\mathrm{T}$ wave abnormalities, the great majority $(85 \%)$ of those in which a cause was traced were due to one of three conditions; namely coronary heart disease, digitalis, or ventricular hypertrophy, and the commonest was coronary disease.

Since slight ST and $\mathrm{T}$ wave abnormalities are most commonly due to coronary heart disease and since they are frequently the only cardiographic abnormalities present in this disease, their importance can hardly be denied. These changes are abnormal when seen in any tracing recorded under standard conditions, and they must therefore be significant. (Routine electrocardiograms are not recorded while the patient is driving through rushhour traffic or drinking iced water.) The logical attitude is therefore to regard definite ST and $T$ wave abnormalities of ischaemic type as being due to coronary heart disease until proved otherwise. Most of the conditions which cause similar abnormalities can be excluded by careful attention to the history and clinical examination. The greatest difficulty in differential diagnosis is presented by left ventricular hypertrophy, and this possibility must always be considered when ST and $T$ wave abnormalities are confined to the lateral leads.

If minor cardiographic changes are to be recognized and evaluated correctly, the record must, as Smith (1968) has emphasized, be of the highest technical quality, and the praecordial electrodes must be accurately placed. A wandering baseline or somatic tremor can obscure minor changes, and there is evidence that an electrocardiograph with an inadequate low frequency response can actually produce artefactual ST segment shifts (Berson and Pipberger, 1966).

Even with a perfect recording, the reporter will sometimes be forced to admit that he cannot be certain whether the appearances are abnormal or not. Slight ST depression associated with tachycardia, or ST depression of the upward sloping (J) type may be impossible to classify. $T$ wave voltage varies so greatly from one individual to another depending on age, body build, and other factors that it may not be possible to classify a single tracing as normal or abnormal. There must therefore be a borderline category, and due allowance must be made for observer variation.

We have now reached a position where we can define the role of the electrocardiogram in the diagnosis of coronary heart disease.

We have shown that though large myocardial infarcts usually exhibit classical patterns which are seldom mimicked by any other condition, small infarcts frequently manifest only ST and/or T wave abnormalities which may be very slight and which are not specific for coronary disease.

Thus, though the cardiogram is by far the most important piece of objective evidence in the diagnosis of coronary disease, it should not be regarded as providing a ready-made solution. The diagnosis must be based on the total evidence, of which the tracing is only a part. The more classical the pattern, the more probable is the presence of infarction, but even the most typical pattern has occasionally misled (Mamlin, Weber, and Fisch, 1964). At the other end of the scale, a normal electrocardiogram cannot be taken as excluding infarction.

Minor ST and $T$ wave abnormalities are of great importance. They should not be disparaged on the ground that they are non-specific, for they are most commonly due to coronary disease and are frequently the only objective evidence of it. The various conditions which give rise to similar abnormalities must be excluded by a careful history and clinical examination. Often this is easy; pregnancy, hypothyroidism, and drugs can readily be eliminated in most cases. But sometimes the exercise is more difficult and requires considerable experience. One of the commonest problems is the interpretation of slight $\mathrm{ST}$ and $\mathrm{T}$ wave abnormalities confined to the lateral leads (Short, 1969). These changes are consistent with both coronary disease and left ventricular hypertrophy. In the latter condition there is commonly left axis deviation and increased QRS voltage, but these features are sometimes absent, particularly in patients with obesity or a deep chest. The two conditions can only be differentiated by careful clinical examination. It is not sufficient to know the patient's blood pressure. The pressure may be raised without causing left ventricular hypertrophy. Conversely, left ventricular hypertrophy may be present without hypertension, as in aortic valve disease or mitral regurgitation; and though these valvular lesions give rise to characteristic murmurs, these are not always obvious, and similar murmurs may be present in the absence of significant valvular disease. An effort test may provide valuable evidence in this dilemma, but it should never be 
undertaken if there is any suspicion of recent infarction.

The most serious limitation of the electrocardiogram is its frequent failure to indicate whether the cardiac lesion is progressive and potentially dangerous, or old and relatively unimportant. This-the most vital step in diagnosis-often demands considerable experience in clinical cardiology. Some idea of the difficulty may be appreciated from the fact that out of 211 suspected slight or subacute coronary attacks investigated clinically, electrocardiographically, and by enzyme studies, only twothirds could be diagnosed with confidence (Short, 1968); in the remainder it was impossible to be certain whether there had been recent infarction or not, and all that could be said was that it was more or less probable. If the cardiogram alone had been available, many mistakes would have been made, for a considerable proportion of the patients who proved to have acute infarction showed only minor cardiographic abnormalities, and some no definite abnormality at all; whereas many of the patients without recent infarction had grossly abnormal cardiograms from previous coronary attacks. Serial electrocardiography is invaluable in this situation; but its role is confirmatory, for if infarction is suspected a diagnosis must be made without delay.

\section{Conclusions}

The role of the electrocardiogram in the diagnosis of coronary heart disease has been considered against the background of the demand for an electrocardiogram reporting service.

While the cardiogram is the most valuable aid to the diagnosis of coronary heart disease, it does not provide a ready-made answer. In particular, it commonly fails to discriminate between a recent and potentially dangerous lesion, and one which is healed and irrelevant to the patient's present symptoms.

Minor abnormalities of the ST segment and T wave, though non-specific, are of great importance. They are most commonly due to coronary disease and are often the only objective evidence of it; furthermore, the conditions which cause similar abnormalities can nearly always be excluded by a careful history and clinical examination.

The cardiogram of a patient with suspected coronary heart disease must be interpreted against the background of the total evidence, and this demands considerable experience in clinical cardiology. Thus, an electrocardiogram reporting service, while it may have a limited role in the diagnosis of arrhythmias and ventricular hypertrophy, is unsatisfactory and potentially dangerous in the diagnosis of coronary disease.
REFERENCES

Berson, A. S., and Pipberger, H. V. (1966). The low-frequency response of electrocardiographs, a frequent source of recording errors. Amer. Heart f., 71, 779.

Bisteni, A., Medrano, G. A., and Sodi-Pallares, D. (1961). Ventricular premature beats in the diagnosis of myocardial infarction. Brit. Heart f., 23, 521.

Blackburn, H., Keys, A., Simonson, E., Rautaharju, P., and Pursar, S. (1960). The electrocardiogram in population studies; a classification system. Circulation, 21, 1160.

British Medical Association (1968). Report of Annual Representative Meeting, Eastbourne, 1968. Brit. med. F., 3, Suppl., p. 19.

Burns-Cox, C. J. (1967). Return to normal of the electrocardiogram after myocardial infarction. Lancet, $1,1194$.

Cook, R. W., Edwards, J. E., and Pruitt, R. D. (1958a). Electrocardiographic changes in acute subendocardial infarction. I. Large subendocardial and large nontransmural infarcts. Circulation, 18, 603.

,-- , and $-(1958 \mathrm{~b})$. Electrocardiographic changes in acute subendocardial infarction. II. Small subendocardial infarcts. Circulation, 18, 613.

Evans, W. (1965). Presaging cardiac pain. Brit. Heart f., 27, 856.

- , and McRae, C. (1952). The lesser electrocardiographic signs of cardiac pain. Brit. Heart $\mathcal{F} ., 14,429$.

Friedberg, C. K. (1966). Diseases of the Heart, 3rd ed., p. 679. W. B. Saunders, Philadelphia.

- , and Zager, A. (1961). "Nonspecific" ST and T-wave changes. Circulation, 23, 655.

Lorimer, A. R., and Kennedy, J. A. (1968). A general practitioner electrocardiogram service. Scot. med.F., 13, 223.

Mamlin, J. J., Weber, E. L., and Fisch, C. (1964). Electrocardiographic pattern of massive myocardial infarction without pathologic confirmation. Circulation, 30, 539.

Mitchell, J. R. A., and Schwartz, C. J. (1963). The relation between myocardial lesions and coronary artery disease. II. A selected group of patients with massive cardiac necrosis or scarring. Brit. Heart f., 25, 1.

Papp, C., and Smith, K. S. (1951). Electrocardiographic patterns in slight coronary attacks. Brit. Heart f., 13, 17.

Seymour, J., Conway, N., and Bridger, E. (1968). Electrocardiograph service for general practitioners: an appraisal. Brit. med. F., 1, 305.

Short, D. (1968). Value and limitations of electrocardiogram in diagnosis of slight and subacute coronary attacks. Brit. med. F., 4, 673.

- (1969). Problems of electrocardiogram reporting in suspected coronary disease. To be published.

Skjaeggestad, O., and Molne, K. (1966). Electrocardiogram in patients with healed myocardial infarction disclosed at autopsy. Acta med. scand., 179, 23.

Smith, K. S. (1968). Electrocardiography misused. Brit. Heart $\mathcal{F} ., 30,1$.

Sodi-Pallares, D., Bisteni, A., Cisneros, F., de Micheli, A., Medrano, G. A., and Testelli, M. R. (1963). Reliability of the electrocardiogram in diagnosis of myocardial infarction. In Coronary Heart Disease: The Seventh Hahnemann Symposium, p. 278. Ed. by W. Likoff and J. H. Moyer. Grune and Stratten, New York.

Woods, J. D., Laurie, W., and Smith, W. G. (1963). The reliability of the electrocardiogram in myocardial infarction. Lancet, $2,265$.

World Health Organization (1959). Hypertension and Coronary Heart Disease: Classification and Criteria for Epidemiological Studies: First Report of the Expert Committee on Cardiovascular Diseases and Hypertension. Wld Hlth Org. techn. Rep. Ser., No. 168. 